\title{
A FRAMEWORK FOR ANALYZING COMBINED LOAD-CARRYING-CAPACITY OF PLUG-IN ELECTRIC VEHICLES AND SOLAR GENERATORS
}

\author{
Soumyo V. Chakraborty ${ }^{*}$, Sandeep K. Shukla ${ }^{*}$, James Thorp
}

\begin{abstract}
$^{1}$
In this paper, we study the combined effect of vehicle-to-grid (V2G) operations and solar PV generation on the power grid in terms of their contribution to effective load carrying capacity (ELCC). Our experiments are based on a multi-phase simulation and optimization framework we have designed and implemented along with actual hourly load data, actual hourly time synchronized solar radiation data, and actual capacity auction market data for New York City, and real projections of the plug-in electric vehicle (PEV) population. This work considers the change in ELCC for varying levels of installed solar generation capacity and size of PEV fleets, and quantifies the economic benefits of these changes utilizing the capacity auction market data. Our study shows a potential V2G ELCC of $200 \mathrm{MW}$ in New York City for the expected number of PEVs in 2015-2017. This represents about \$20 million in economic benefit per year from capacity gains alone. About $60 \%$ of the battery costs are recoverable from the PEV capacity contributions at initial PEV penetration levels. A unique observation from our study is that, contrary to conventional expectation, there is no marginal gain in solar ELCC from PEVs above the ELCC we already obtain from the PEVs alone.
\end{abstract}

\section{Key Words}

Plug-in electric vehicles, vehicle-to-grid, renewable generation, solar energy, effective load carrying capacity, commute profile

\section{Introduction}

The penetration of electric cars is expected to grow at a rapid pace over the current decade with the projected number of electric vehicles on the road in the US alone being in the 1 million range by 2015-2017 [1]. At the same time, installed solar generation capacity in the US has been roughly doubling each year for the last few years with the trend expected to continue [2]. In this paper, we have analyzed the combined impact of increasing penetrations of plug-in electric vehicles (PEVs) and solar generators on the load carrying capacity of the grid.

PEVs can impact an electric grid via vehicle-to-grid (V2G) and grid-to-vehicle (G2V) operations. For our study, we consider PEVs only as those are the vehicles that connect to the electric

\footnotetext{
${ }^{1}$ This is a revised and expanded version of the paper titled "A Framework For Analyzing Load-Carrying-Capacity Of Plug-In Electric Vehicles And Impact On Solar Generators" by the same authors presented at the 2011 IASTED European Conference on Power and Energy Systems, Crete, Greece * Department of Electrical and Computer Engineering, Virginia Polytechnic Institute and State University, Blacksburg, VA 24061, USA; e-mail:\{soumyo, shukla, jsthorp\} @vt.edu

Recommended by Prof. Z. Salameh

(DOI: 10.2316/Journal.215.2013.2.215-1050)
}

grid for charging their batteries via G2V operation, and, potentially supply energy back to the grid from their battery if necessary which is commonly known as the V2G operation. PEVs encompass both pure electric vehicles known as Battery Electric Vehicles (BEV) as well as the Plug-in Hybrid Electric Vehicles (PHEV).

On one hand, charging of the electric vehicle batteries (G2V) can represent significant additional load on the grid. If the timing of this charging is not properly managed, it may lead to spikes in peak load reducing the reliability of a grid unless additional generation capacity is added. Also, spatial localization of vehicle charging spots can cause bottlenecks in the electricity distribution network. On the other hand, given the potential for V2G, the electric vehicles represent a significant amount of distributed storage capacity available to the grid. If the timing of vehicle charging and discharging (to the grid via V2G) are properly optimized, the fleet of electric vehicles can be harnessed to effect temporal shifting of load from peak hours to non-peak hours with the vehicles effectively acting as a negative load during peak hours by supplying electricity back to the grid. This can contribute additional load carrying capacity to the grid. This capacity can be measured by effective load carrying capacity (ELCC). ELCC of an energy source is defined as its ability to support additional peak load without increasing the grid's loss of load probability (i.e. the probability that generation will be less than load at some point over a time window) [3]. Alternatively, ELCC of a source represents the amount of existing supply capacity that can be removed by adding the source while serving the same peak load without increasing the loss of load probability.

In this study, we have developed a practical optimization algorithm for the V2G control and built a simulation framework to analyze the effective load carrying capacity added to a grid by different populations of electric vehicles. We ran this simulation framework utilizing actual hourly load data of New York City for the full years of 2008 and 2009 and with the projected electric vehicle penetration numbers for the city in 2015-2017 [1]. Due to ongoing energy efficiency initiatives, we assumed the load pattern in the 2015-2017 timeframe will be reasonably close to the current one. New York City was chosen for testing the optimization algorithm and simulation framework because (1) it is the largest metropolitan area in the United States, (2) it is projected to be one of the early adopter cities for electric vehicles, and (3) it has a well established price history for electric capacity via the auctions market. For battery capacity and mileage, we used the official specifications released by the US Environmental Protection Agency (EPA) based on their actual testing of Chevy Volt 
[4].

We have obtained promising initial results from our study. We observe that the incremental load carrying capacity contributed by V2G increases steadily with the size of the fleet. Capacity auction market data has been used to quantify the economic benefit contributed by the PEVs due to their load carrying capacity. Our results show a potential PEV ELCC of 200 MW in New York City for the expected number of PEVs in 2015-2017. This represents about \$20 million in economic benefit per year from capacity gains alone. Our estimation model demonstrates that at smaller fleet sizes, up to $60 \%$ of the projected battery costs of electric vehicles are recovered via the load carrying capacity contributed to the grid alone. At projected level of fleet size for 2015-2017, about a third of the cost is recovered. This represents a compelling economic argument for the electric vehicles aside from the environmental benefits or energy security benefits that accrue from the electric vehicles. We also believe that, based on these results, a policy argument can be made for grid operators to share a portion of the economic benefit from the incremental load carrying capacity toward helping finance part of the cost of electric cars.

The requirements of charging the PEV batteries via G2V operations can increase the aggregate load in the grid. However, in a related research [5], we had shown that at the PEV penetration levels under study, the grid's capacity to charge the PEVs is more than adequate using the scheme of charging the PEVs during off hours.

We further augmented our simulation framework to analyze the impact of electric cars on the effective load carrying capacity of solar generators to identify what kind of enhancement, if any, can be obtained to the solar ELCC. For running the simulation, we utilized actual hourly solar radiation data set from 2008 and 2009 for the New York area synchronized with the hourly load data. The results from this simulation are also quite insightful. The study shows that there is no demonstrable marginal gain in solar ELCC from electric vehicles on top of the ELCC we already obtain from the electric vehicles alone. So, contrary to conventional expectation, we did not observe an economic benefit for solar generators attributable to the electric vehicles.

Upon closer analysis of the behavior of combined ELCC of solar generators and PEVs as the installed capacity of solar generators increases, we observed a gradual reduction in the incremental ELCC contributed by PEVs at higher levels of solar penetration. As part of this research, we devised an approach to mitigate this loss by temporal spreading of PEV commute profiles. Based on simulation studies, our proposed temporal mitigation approach yields up to $72 \%$ improvement in an overall ELCC metric for PEVs and solar generators.

\section{Previous Work}

Kempton and Letendre [6] discussed the possibility of plug-in electric vehicles as a power source in 1997. There has been several studies since then involving grid-connected PEVs [7-10] particularly focusing on the V2G operations and their economic benefits [11-13]. Potential economic impacts of large-scale V2G operations were analyzed in [14]. An estimation of potential emissions reductions due to PHEVs in Colorado under various charging scenarios is provided in [15]. Case studies for V2G economic analysis for Sweden and Germany were done in [16]. An actual project in Denmark focusing on the integration of electric vehicles into a smart grid and their control strategies is described in [17]. A simulation-based estimation of power system regulation services from V2G operations in Western Denmark with high wind energy penetration is described in [18]. An analysis of leveraging aggregated plug-in electric vehicle loads to provide regulation service to a grid via demand dispatch and utilization of Google's smart charging algorithm for electric vehicles can be found in [19]. Communication architecture and protocols for linking the electric vehicles, charging stations, power generating units, the grid and control points are discussed in $[20,21]$.

A survey of various approaches for computing the capacity credit of power generating units and their application for conventional power plants and wind power generators are described in $[22,23]$. The Task Force on the Capacity Value of Wind Power of the IEEE Power and Energy Society identified a preferred capacity calculation method for intermittent energy sources based on ELCC [24] which has been adopted in this paper.

Details of a study of the ELCC of solar photovoltaic generators under different solar panel geometries and varying levels of solar penetration on a state-by-state basis in the United States are provided in [25].

In none of the previous work we examined, we found the type of quantification of the combined ELCC contributions of PEVs and solar generators as we report in this paper, nor did we encounter a flexible simulation framework that analyzes the impact of temporal adjustments in commute profiles on grid ELCC in a systematic way.

\section{Background}

We consider the perspectives of three primary stakeholders in this study:

(1) grid operators/utilities

(2) owners of electric vehicles, and

(3) solar power generators

PEVs represent a significant new load for the electric grid. At the most common charging level envisioned (Level 2 charging) of $240 \mathrm{~V}$ x 30A [1], each PEV will add $7.2 \mathrm{~kW}$ of additional load while charging. This can add up to $400 \mathrm{MW}$ to the peak demand in New York City by 2015-2017 based on the PEV fleet projections [1] if charging is not controlled by a suitable optimization scheme. From the point of view of grid owners or utilities, serving this additional peak demand will require provisioning of expensive additional capacity assuming that the reliability of the grid (i.e. loss of load probability) cannot be compromised.

From the point of view of the vehicle owners: (1) For PHEVs (e.g. Chevy Volt), the miles driven using electricity have to be maximized (and miles driven using gasoline minimized) in order for them to realize the full economic and environmental benefit. So it is desirable to have enough charge in the battery that can last until the next re-charging. (2) For BEVs (e.g. Nissan Leaf), running on gasoline is not even an option as these cars do not have an internal combustion engine. So there always has to be enough charge left in the battery before the next re-charging for the owners to avoid getting stranded on the road.

Solar power generators face the issue of variability and uncertainty related to their power output due to the variable and unpredictable nature of sunlight. This intermittency in power output results in a relatively low ELCC of solar generators. A prior study found the ELCC of solar photovoltaics in New York to be in the $28 \%$ to $53 \%$ range for solar penetration ranging from 
$20 \%$ to $2 \%$ [25]. Having adequate energy storage capabilities available might allow the solar generators to smooth out the variability in their power output to some degree potentially improving their ELCC.

\section{Proposed Framework}

We devised an optimization algorithm for charging (G2V) and discharging (V2G) of PEVs with a view toward efficacy and ease of implementation in practice. The algorithm schedules the vehicles to charge in the off-peak hours after their owners have driven back home from work. This is to ensure that the vehicles do not increase the peaking capacity requirement of the grid. During the working hours, the vehicles are plugged into the grid while parked and kept on a stand-by mode for potential V2G operation. If the supply capacity of the grid falls below the aggregate load at any point during those hours, the grid connected vehicles will try to meet that shortfall by supplying power back into the grid from their batteries as long it will not cause the charge level in the batteries to drop below the minimum level of charge required for the cars to be driven back from work to home. So, the objective of the algorithm is to minimize the loss of load in the grid while satisfying the required state of charge criteria of the electric vehicle owners. A high-level outline of the optimization algorithm is provided in Figure 1.

Our Monte Carlo-based simulation framework has four phases.

Phase 1: Actual aggregate load on the grid at hourly frequency (or any other frequency chosen) is played back into the framework. It also simulates the total power supply available to the grid from all the generating units combined at each of the sampling intervals taking into account the failures of individual generating units. As an example, we have taken the peak supply capacity of a grid to be the peak load over a number of years plus a reserve margin. We have used statistical simulation to derive a model of hourly failure rates of the individual generating units based on the actual load time series data, peak supply capacity and the required reliability (i.e. the target loss of load probability).

Phase 2: We then introduced gradually increasing numbers of PEVs into the simulation framework with their V2G operations controlled by our V2G optimization algorithm. As the PEVs are introduced, we took off some of the existing generating capacity from the grid to observe if there is degradation in the grid reliability - i.e. if there is an increase in the loss of load probability. Amount of existing generating capacity that can be taken off without impacting the grid reliability represents the ELCC of the given fleet of PEVs.

Phase 3: We subsequently re-ran the simulation framework by introducing gradually increasing solar generation capacity but without the PEVs. We played back the actual solar irradiation numbers into the framework for the same region as that served by the grid. The solar radiation numbers are time-synchronized with the load figures. We then used the solar radiation numbers to simulate the power output from a collection of solar photovoltaic generators using a linear function (since output of solar photovoltaics is proportional to the incident solar irradiation). As the solar capacity is introduced, we took off some of the existing generating capacity from the grid to observe if there is a degradation in the grid reliability. Amount of existing generating capacity that can be taken off without impacting the grid reliability represents the ELCC of the given set of solar generators without any V2G operations.

Phase 4: In the last phase of the simulation, we re-introduced the PEVs and controlled their V2G operation using our V2G optimization algorithm. We re-ran the simulation to re-compute the ELCC of the varying sizes of solar capacity with V2G operations present in the grid.

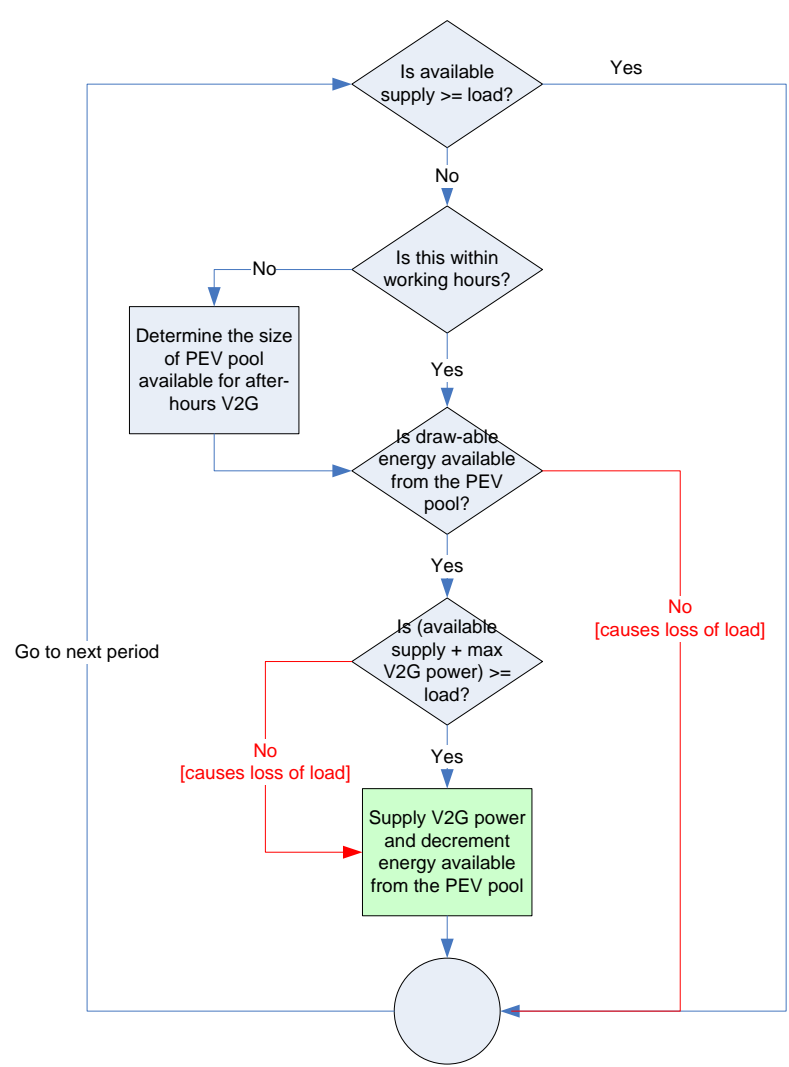

Figure 1: A high-level outline of our proposed V2G optimization algorithm.

More formally, let us assume, we have the following relevant variables:

$L$ - pattern of aggregate load on the grid over a time pe$\operatorname{riod} \mathrm{T}$

$s$ - aggregate peak supply capacity

$f$ - model-based hourly failure rate of existing individual generating units

lolp - reliability of the grid, represented by the loss of load probability

$B$ - effective operating range of the state of charge of a PEV's battery

$c$ - average length of commute in miles

$m$ - mileage of the PEV in miles per Wh

$P$ - charging/discharging rate of the PEV battery

$I$ - after-hours V2G participation rate of PEVs

$R$ - pattern of solar radiation in the region under study over time period $\mathrm{T}$

Note that the variables in capital letters represent vectors and the ones in lower case letters represent scalar values. $f_{1}, f_{2}, g_{1}, g_{2}$ in the equations below represent the functions being simulated.

Relationships represented by these functions are complex and do not necessarily have analytical forms. The equations 
below outline the key independent and dependent variables involved in the relationships. However, actual relationships are simulated via code in our simulation framework.

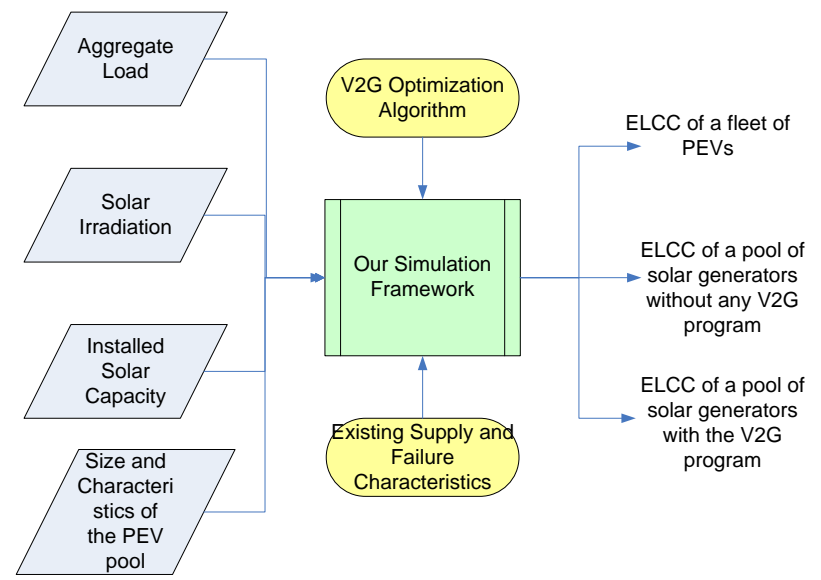

Figure 2: Key input and output components of the proposed simulation framework.

$$
\text { lolp }=f_{1}(L, s, f)
$$

In Phase 1 of the simulation, we calibrate $f$ so we obtain the target lolp given $L$ and $s$.

In Phase 2 of the simulation, for each run i, we introduce $n_{i}$ number of PEVs participating in the V2G program, and subtract generating capacity $e p_{i}$ from the existing capacity $s$. The goal is to come up with an $e p_{i}$ such that the grid's loss of load probability remains unchanged at lolp.

$$
\text { lolp }=f_{2}\left(L, s-e p_{i}, f, n_{i}, B, c, m, P, I\right)
$$

So $e p_{i}$ represents the ELCC of a fleet of PEVs of size $n_{i}$ (for given values of $B, c, m, P$ and $I$ ).

In Phase 3 , for each run $\mathrm{j}$, we introduce total solar photovoltaic rated capacity $v_{j}$ into the grid and subtract generating capacity $e_{j}$ from existing capacity $s$. Note that at this phase we assume there is no V2G program available in the grid. The goal is to come up with an $e s_{j}$ such that the grid's loss of load probability remains unchanged at lolp.

$$
\text { lolp }=g_{1}\left(L, s-e s_{j}, f, v_{j}, R\right)
$$

So $e s_{j}$ represents the ELCC of a pool of solar generators with rated capacity $v_{j}$ without any $\mathrm{V} 2 \mathrm{G}$ program in the grid.

Finally, in Phase 4, we re-run each iteration j from Phase 3 after introducing $n$ number of PEVs participating in the V2G program. Generating capacity $e s p_{j}$ is subtracted from existing capacity $s$. Again, the goal is to come up with an $e s p_{j}$ for each $v_{j}$ such that the grid's loss of load probability remains unchanged at lolp.

$$
\text { lolp }=g_{2}\left(L, s-e s p_{j}, f, v_{j}, R, n, B, c, m, P, I\right)
$$

So $e s p_{j}$ represents the ELCC of a pool of solar generators with rated capacity $v_{j}$ with a V2G program in the grid that includes $n$ PEVs.

Let $\Delta e s_{j}$ represent the gain in solar ELCC for rated solar capacity of $v_{j}$ that is attributable to the V2G operations involving a fleet of $n$ PEVs.

$$
\Delta e s_{j}=e s p_{j}-e s_{j}
$$

Let's assume ep represents the ELCC of $n$ PEVs participating in the V2G program from (2).

The maximum marginal net ELCC accrued to solar generators due to a V2G program involving $n$ PEVs is given by $\Delta e$.

$$
\Delta e=\max \left(\Delta e s_{j}\right)-e p
$$

\section{Experiments}

We obtained hourly aggregate load data for New York City for all of 2008 and 2009 from the New York Independent System Operator records [26]. For calculating peak supply capacity of the grid, we added the requisite reserve margin [27] on top of the peak load from 2008-2009. Loss of load probability is obtained from reliability guidelines of the New York State Reliability Council [27]. Projected size of the PEV fleet in New York City, their charging/discharging rates, and average commute miles per day were obtained from [1]. We used the battery capacity, effective operating range of the battery's state of charge, and number of Wh consumed to drive a mile for Chevy Volt from the official specifications released by the Environmental Protection Agency [4]. Hourly solar radiation data for all of 2008 and 2009 for the New York area were obtained from the National Climatic Data Center [28].

All these data were used as inputs to our optimization algorithm and simulation framework to obtain the ELCC figures under different scenarios.

\section{Results}

As we increased the size of PEV fleet participating in V2G, we saw a gradual increase in the ELCC contributed to the grid by the PEVs as shown in Figure 3.

For the projected number of PEVs in New York City [1] in 2015/2017 - 54,069 - the ELCC is 200 MW assuming all the PEVs participate in V2G.

Based on additional simulations we ran for a subsequent study [5], we were able to confirm that at these levels of PEV penetration, G2V power does not become a constraint, and there is always adequate grid capacity to fully charge the PEV batteries during off hours.

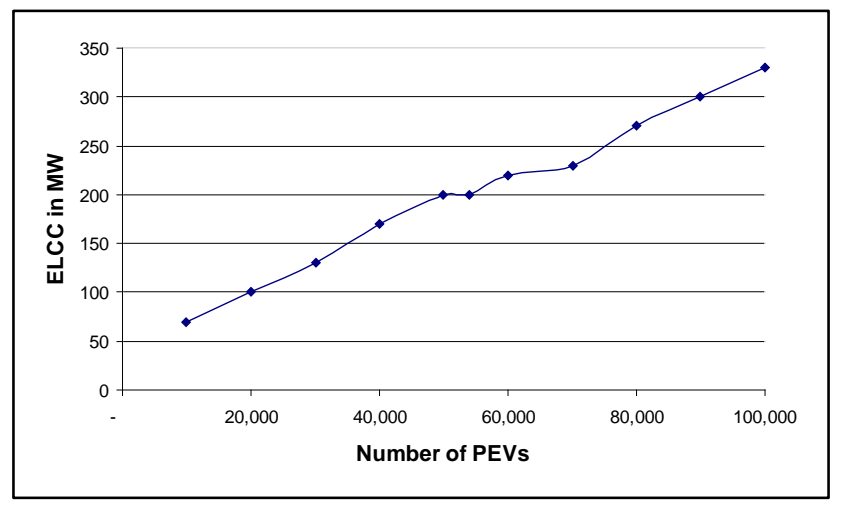

Figure 3: ELCC of PEV fleets of various sizes. 
When we ran the simulations with increasing amounts of installed solar photovoltaic generating capacity, we observed the pattern of solar ELCC shown in Figure 4 without any V2G program present in the grid.

As evidenced from Figure 4, solar ELCC seems to flatten out at around $700 \mathrm{MW}$ without any presence of V2G operation in the grid.

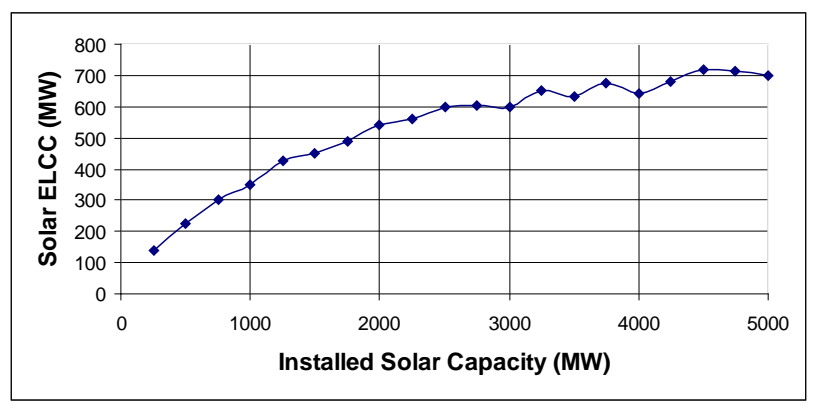

Figure 4: ELCC of solar generation without V2G.

In percentage terms, ELCC of solar generation gradually drops from a high of 55\% of installed capacity at low levels of solar penetration to around $14 \%$ of installed capacity at high penetration levels. The details of solar ELCC in \% terms are given in Table 1 . This result seems consistent with $53 \%$ solar ELCC reported earlier for New York state at low levels of solar penetration [25].

With the projected number of PEVs in 2015-2017 [1], solar ELCC gets a significant boost at lower levels of solar penetration. Comparing Figures 4 and 5, we observe that solar ELCC with V2G starts at $338 \mathrm{MW}$ for $250 \mathrm{MW}$ of installed solar capacity as opposed to $138 \mathrm{MW}$ of ELCC without V2G. But ELCC appears to flatten out at around $750 \mathrm{MW}$ as seen from Figure 5 assuming all the PEVs participate in V2G. Table 2 shows the ELCC of solar generation as a \% of total installed capacity for varying levels of installed capacity with V2G operation present in the grid for the projected number of PEVs in 2015-2017.

Figure 6 shows the trend in solar ELCC with and without V2G. As evident from the chart, at low levels of solar capacity, there is a significant difference in ELCC between the two scenarios, e.g. 135\% with V2G versus 55\% without V2G for 250 MW of installed capacity. Our observation about the greater than $100 \%$ solar ELCC with V2G at low levels of installed solar capacity is that it is due to the ELCC contributed by the PEV fleet alone (200 MW) which has been counted in the solar ELCC figure. V2G contributed boost in solar ELCC diminishes gradually as solar capacity grows. There is virtually no gain in ELCC as we reach around $5000 \mathrm{MW}$ of installed capacity.

Finally we subtracted the solar ELCC without V2G from solar ELCC with V2G, based on (5), at different levels of installed solar capacity to identify the gain in solar ELCC that is attributable to V2G. Figure 7 shows the trend in ELCC gain as the installed capacity increases. The gain starts out at $200 \mathrm{MW}$ at low levels of solar capacity and goes down to around $50 \mathrm{MW}$ as the capacity reaches $5000 \mathrm{MW}$. The important result here is that the ELCC of the V2G operation alone (without any solar capacity) is $200 \mathrm{MW}$ at the projected size of the PEV fleet. So, in effect, we see no marginal net gain in ELCC of solar generation, per (6), that is attributable to PEVs and which cannot be obtained by the V2G operation alone.
Table 1

ELCC of Solar Generation (\%) of Installed Capacity without V2G

\begin{tabular}{|c|c|}
\hline $\begin{array}{l}\text { Installed Solar Capacity } \\
\text { (MW) }\end{array}$ & $\begin{array}{c}\text { Solar ELCC } \\
\text { (\%) }\end{array}$ \\
\hline 250 & 55 \\
\hline 500 & 45 \\
\hline 750 & 40 \\
\hline 1000 & 35 \\
\hline 1250 & 34 \\
\hline 1500 & 30 \\
\hline 1750 & 28 \\
\hline 2000 & 27 \\
\hline 2250 & 25 \\
\hline 2500 & 24 \\
\hline 2750 & 22 \\
\hline 3000 & 20 \\
\hline 3250 & 20 \\
\hline 3500 & 18 \\
\hline 3750 & 18 \\
\hline 4000 & 16 \\
\hline 4250 & 16 \\
\hline 4500 & 16 \\
\hline 4750 & 15 \\
\hline 5000 & 14 \\
\hline
\end{tabular}

\section{Analysis of Results and a Potential ELCC Enhancement Approach}

One of the possible reasons why we saw the marginal gain in ELCC contributed by PEVs go down with increasing installed solar capacity is the fact that as installed solar capacity goes up it is able to meet more demand during the day-time working hours that was earlier met by energy from the PEV batteries via V2G operations. As a result, overall system ELCC is not improved significantly. We hypothesized that by temporal spreading of daily commute times and hence the V2G availability windows, we would be able to address power shortfalls over a longer period of time during the day and, in the process, would improve the overall system ELCC.

To validate our hypothesis, we designed an experiment where we used a series of different daily commute profiles and computed the combined ELCC of solar generators and PEVs for different levels of installed solar capacity under these various commute scenarios.

The commute scenarios we used in our experiments are shown in Table 3. For each scenario, the fraction of PEVs that start commute (either to or from work) at each hour is shown in the corresponding cell. For example, for Scenario-9, 60\% of vehicles start commute to work at $8 \mathrm{am}, 20 \%$ start commute to work at $9 \mathrm{am}$ and $20 \%$ start commute to work at $10 \mathrm{am}$. Similarly, for the same scenario, $60 \%$ of PEVs start commute from work to home at $5 \mathrm{pm}$ (i.e. hour 17), 20\% start commute form work at $6 \mathrm{pm}$ and $20 \%$ start commute from work at $7 \mathrm{pm}$. We assume that commute takes an hour each way during which time PEVs are not 
available for V2G. PEVs are available for V2G while plugged in at work.

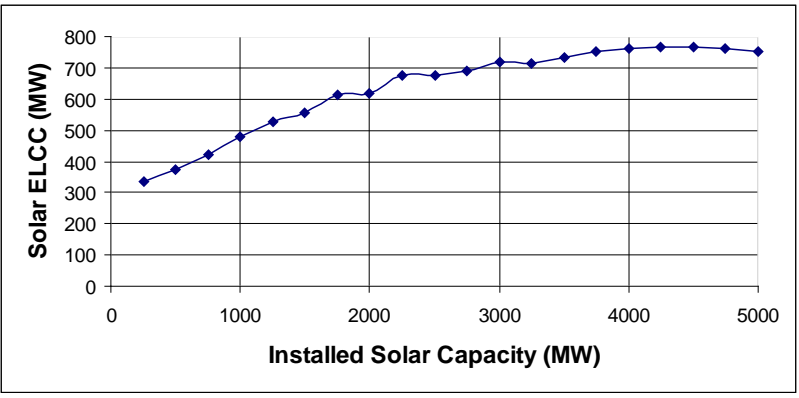

Figure 5: ELCC of solar generation with V2G.

Table 2

ELCC of Solar Generation (\%) of Installed Capacity with V2G

\begin{tabular}{|c|c|}
\hline $\begin{array}{l}\text { Installed Solar Capacity } \\
\text { (in MW) }\end{array}$ & $\begin{array}{l}\text { Solar ELCC } \\
\quad \text { (in \%) }\end{array}$ \\
\hline 250 & 135 \\
\hline 500 & 75 \\
\hline 750 & 56 \\
\hline 1000 & 48 \\
\hline 1250 & 42 \\
\hline 1500 & 37 \\
\hline 1750 & 35 \\
\hline 2000 & 31 \\
\hline 2250 & 30 \\
\hline 2500 & 27 \\
\hline 2750 & 25 \\
\hline 3000 & 24 \\
\hline 3250 & 22 \\
\hline 3500 & 21 \\
\hline 3750 & 20 \\
\hline 4000 & 19 \\
\hline 4250 & 18 \\
\hline 4500 & 17 \\
\hline 4750 & 16 \\
\hline 5000 & 15 \\
\hline
\end{tabular}

Using Scenario-1 as the base case, ELCC of PEVs only (with no solar capacity) is $280 \mathrm{MW}$ for the projected number of PEVs in 2015-2017. By adding the ELCC of solar generators only (with no PEVs) for various levels of installed solar capacity, we get the upper limit of combined solar and PEV ELCC as shown in Table 4.

Combined ELCC of solar generators and PEVs that we were able to compute using our framework under various commute profiles are shown in Figure 8. The upper limit of possible combined solar and PEV ELCC for different levels of installed solar capacity (from Table 4) is shown by the thick red line in the graph. We observe that for commute patterns that are more concentrated toward the 9am-5pm work schedule (e.g. scenarios 1-4), com- bined ELCC is much below the potential maximum. As commute profiles are stretched out more and more after 9am, i.e. as more PEVs start their commute to work later and as a result return home from work later, we see noticeable improvement in the overall ELCC. Among the scenarios we studied, the best ELCC performance is produced by Scenario-11 that evenly distributes the commute start time between $8 \mathrm{am}$ and 12 noon with $20 \%$ of PEVs performing their commute during each hour within that time window. A possible factor driving this behavior is the fact that, in Scenario-11, as commute start times are evenly distributed over 5 hours starting at $8 \mathrm{am}$, the vehicle return times are also even distributed over 5 hours starting at $5 \mathrm{pm}$. This ensures a much better V2G coverage during evening hours as more PEVs are parked at their work locations and available for V2G. Better V2G coverage results in more reliable power system performance (i.e. higher ELCC) as the PEVs can supply power in case evening load spikes up.

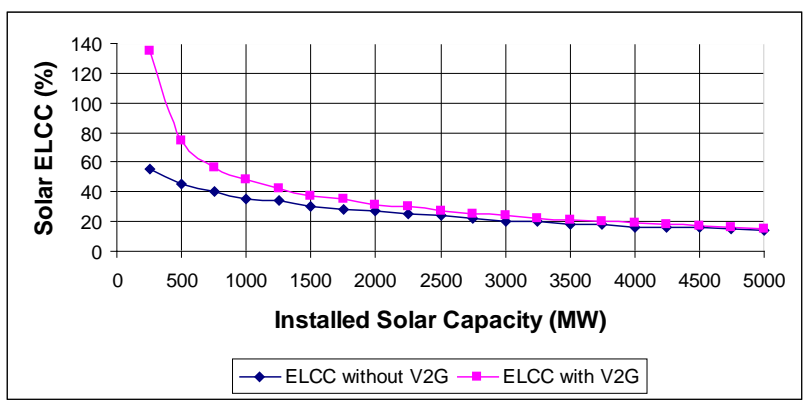

Figure 6. ELCC of solar generation (\%) of installed capacity with and without V2G.

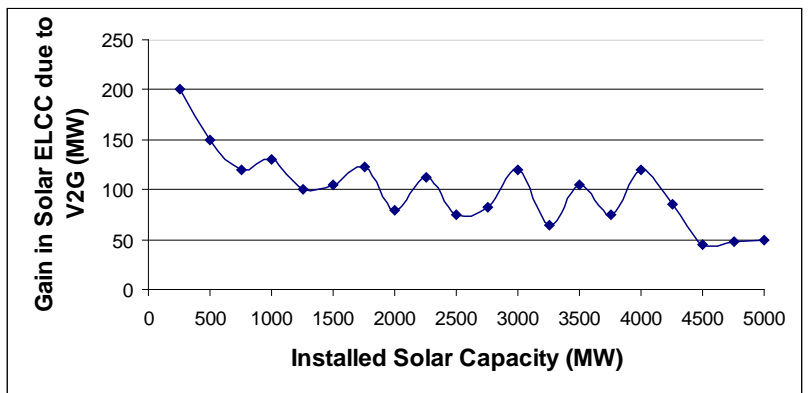

Figure 7. Gain in solar ELCC due to V2G at varying levels of installed solar capacity.

To quantify the overall gain in ELCC due to the temporal spreading in commute profiles, we came up with a metric that represents the loss in combined solar and PEV ELCC with respect to the maximum potential value under different commute scenarios.

$e p_{n}=$ ELCC of a pool of $n$ PEVs without any solar generation from (2)

$e s_{v}=$ ELCC of a pool of solar generators with rated capacity $\mathrm{v}$ without any PEVs from (3)

$$
E_{n, v}=e p_{n}+e p_{v}
$$


$E_{n, v}$ represents the maximum possible combined ELCC from solar generators of capacity $v$ and $n$ PEVs. by,

Loss in combined ELCC from maximum potential is given

$$
C_{n, v}=E_{n, v}-e s p_{v}
$$

where $e s p_{v}$ represents combined solar and PEV ELCC from (4).

We created a metric to compute the overall loss in ELCC from maximum potential under a commute scenario for different levels of installed solar capacity given $n$ PEVs in the grid.

under a given commute scenario

We computed the value of for scenarios 1 through 11 as shown in Figure 9.

Table 3

Different Commute Profiles Showing the Fraction of PEVS Starting Commute at a Given Hour (to/from work)

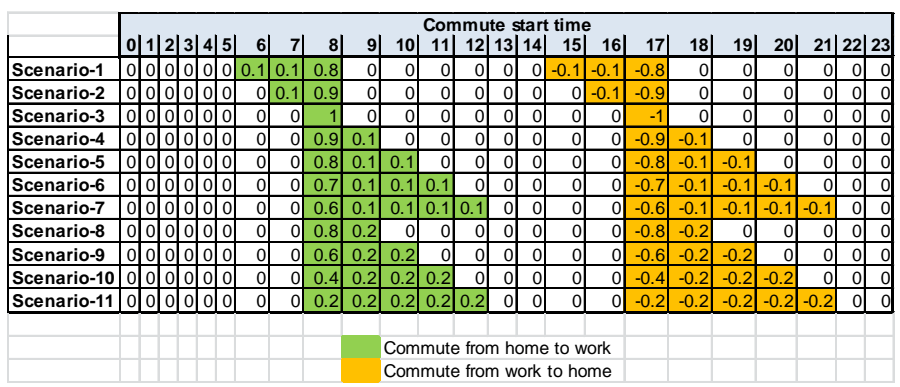

Table 4

Maximum Possible Combined ELCC from Solar and PEVs (MW)

\begin{tabular}{|c|c|c|c|}
\hline Installed Solar Capacity & ELCC PEV Only & ELCC Solar Only & ELCC (Solar Only + PEV Only) \\
\hline 500 & 280 & 180 & 460 \\
\hline 750 & 280 & 275 & 555 \\
\hline 1000 & 280 & 375 & 655 \\
\hline 1250 & 280 & 400 & 680 \\
\hline 1500 & 280 & 425 & 705 \\
\hline 1750 & 280 & 500 & 780 \\
\hline 2000 & 280 & 525 & 805 \\
\hline 2250 & 280 & 540 & 820 \\
\hline 2500 & 280 & 580 & 860 \\
\hline 2750 & 280 & 610 & 890 \\
\hline 3000 & 280 & 625 & 905 \\
\hline 3250 & 280 & 625 & 905 \\
\hline 3500 & 280 & 625 & 905 \\
\hline 3750 & 280 & 630 & 910 \\
\hline 4000 & 280 & 675 & 955 \\
\hline 4250 & 280 & 675 & 955 \\
\hline 4500 & 280 & 680 & 960 \\
\hline 4750 & 280 & 720 & 1000 \\
\hline 5000 & 280 & 720 & 1000 \\
\hline
\end{tabular}

As evidenced from the figure, loss in ELCC is reduced as commute profiles are more spread out over time and they are shifted to the right (i.e. more and more PEVs start their daily commute to work after 9am). Among the scenarios studied, Scenario 11 has the most spread out commute pattern with $20 \%$ of the PEVs scheduled to start their commute each hour between 9am and 12 noon. This also offers the best ELCC performance producing the minimum value to . The primary reason is that this scenario provides good V2G coverage during early evening hours of high system load when solar power output also drops off with the sunset.

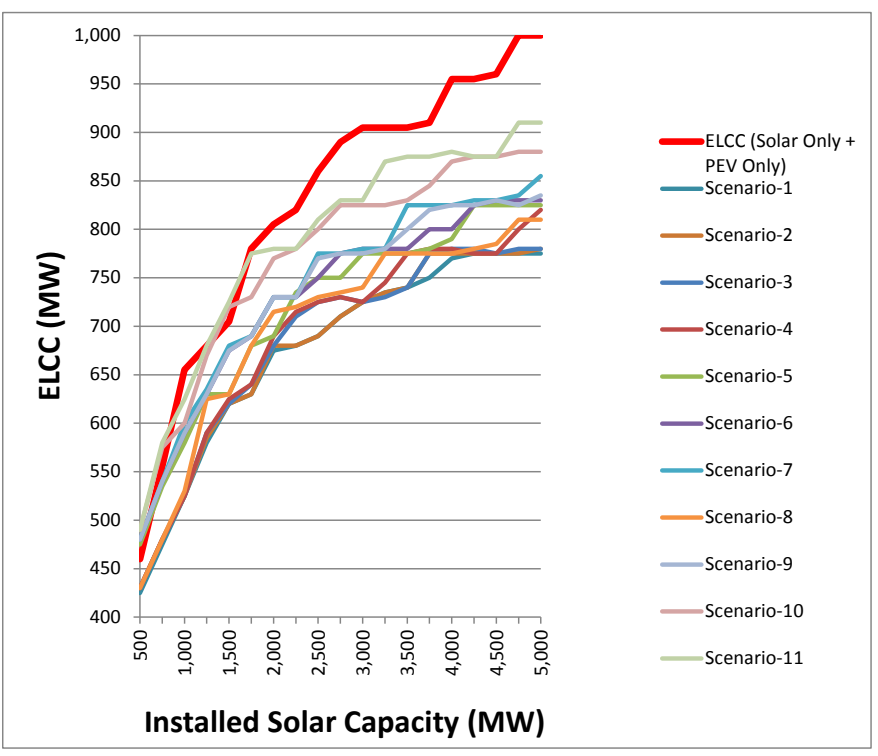

Figure 8: Combined solar and PEV ELCC at varying levels of installed solar capacity under different commute profile scenarios.

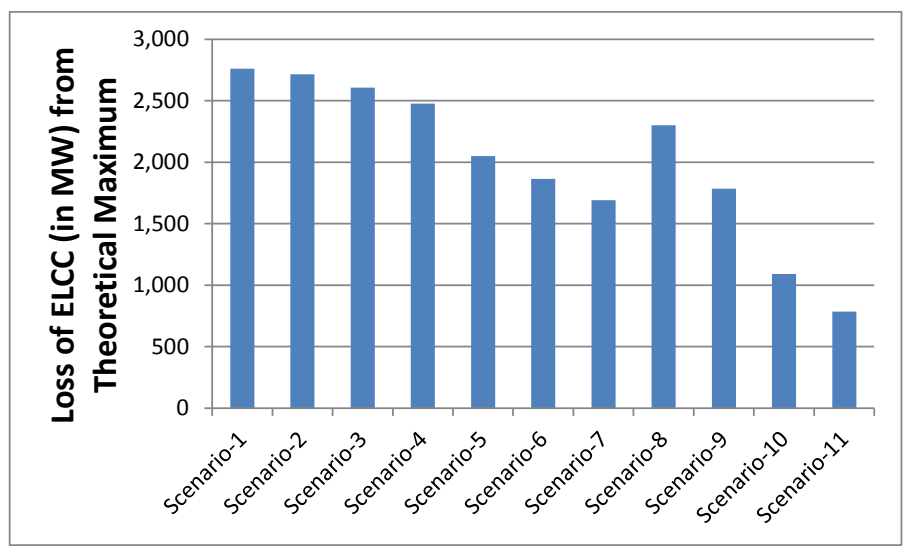

Figure 9: Overall loss of ELCC for each commute scenario compared to the maximum potential value.

\section{Economic Analysis}

We used the results obtained from our framework to estimate the economic benefit derived from PEVs in terms of additional ELCC contributed to the grid. We contrasted this benefit to the projected cost of the PEV battery storage to come up with an analysis of what percentage of the storage costs are recoverable via capacity contributions. According to Gartner Research, the total battery system installed cost is currently around $\$ 900$ per $\mathrm{kWh}$ and falling at $10-15 \%$ per year to reach around $\$ 470$ per $\mathrm{kWh}$ in 2015. Economic life of the PEV batteries was taken to be 8 years based on the battery warranty of Chevy Volt [29] and ignoring any impact on battery life due to V2G operations. Economic value of electric capacity in New York City in the range of $\$ 100$ per $\mathrm{kW}$-yr was arrived at by analyzing the trend in capacity auction prices for the New York City market as recorded by the Federal Energy Regulatory Commission [30]. Based on 
these inputs, we observe the battery storage cost and capacity-related economic benefits from the PEVs as shown in Table 5 in the context of New York City.

We observe that about $60 \%$ of the battery storage costs are recoverable from additional load carrying capacity contributed by the PEVs participating in V2G with fleet sizes in the 10,000 range. For the projected fleet size in 2015-2017 (the highlighted row in Table 5), about a third (32\%) of the storage costs are recovered assuming that all the PEVs participate in V2G. This represents an economic value of \$20 million per year.

By applying our temporal spreading approach of PEV commute profiles, we are able to generate significant enhancement in overall ELCC contribution of PEVs and solar generators. For the projected number of PEVs in 2015-2017 and 5,000 MW of installed solar capacity, by moving from Scenario 1 commute profile to Scenario 11, combined ELCC is improved by $135 \mathrm{MW}$. This represents an additional economic benefit of $\$ 13.5$ million per year.

Several studies [31-32] have looked into the degradation of PEV battery capacity due to frequent discharging related to V2G operations. A multi-variate regression analysis [31] that modeled battery capacity fade as a function of driving-related discharge as well as V2G-related discharge (constant rate) found the relation to be almost linear $\left(\mathrm{R}^{2}=0.96\right)$. The size of the linear regression coefficients imply that battery usage associated with driving has a bigger impact on battery degradation than the loss due to V2G use. In our simulation with target number of PEVs in 2015 in New York City, V2G energy discharge per year per vehicle was roughly $17.2 \mathrm{kWh}$. This represents a capacity-normalized energy of 1.076 (Chevy Volt capacity $=16 \mathrm{kWh}$ ). Using the linear regression coefficient [31] of -0.0000271 for V2G energy discharge, capacity fade due to V2G per year is about $0.003 \%$. Even over the expected life (8 years) of the battery, the total capacity degradation due to V2G appears to be minimal (0.023\%).

Table 5

Economic Gain from Capacity Contributions of PEVs vs. Battery Storage Cost

\begin{tabular}{|r|r|r|c|c|c|}
\hline $\begin{array}{c}\text { Number of } \\
\text { PEVs }\end{array}$ & $\begin{array}{c}\text { Total Storage } \\
\text { Capacity } \\
\text { (in kWh) }\end{array}$ & $\begin{array}{c}\text { Annualized } \\
\text { Storage Cost }\end{array}$ & $\begin{array}{c}\text { ELCC } \\
\text { (in MW) }\end{array}$ & $\begin{array}{c}\text { Value of } \\
\text { Capacity } \\
\text { Gained }\end{array}$ & $\begin{array}{c}\text { Capacity Gain } \\
\text { I Storage Cost }\end{array}$ \\
\hline 10,000 & 160,000 & $11,635,080$ & 70 & $\$ 7,000,000$ & $60 \%$ \\
20,000 & 320,000 & $23,270,161$ & 100 & $\$ 10,000,000$ & $43 \%$ \\
30,000 & 480,000 & $34,905,241$ & 130 & $\$ 13,000,000$ & $37 \%$ \\
40,000 & 640,000 & $46,540,322$ & 170 & $\$ 17,000,000$ & $37 \%$ \\
50,000 & 800,000 & $58,175,402$ & 200 & $\$ 20,000,000$ & $34 \%$ \\
54,069 & 865,104 & $62,909,716$ & 200 & $\$ 20,000,000$ & $32 \%$ \\
60,000 & 960,000 & $69,810,482$ & 220 & $\$ 22,000,000$ & $32 \%$ \\
70,000 & $1,120,000$ & $81,445,563$ & 230 & $\$ 23,000,000$ & $28 \%$ \\
80,000 & $1,280,000$ & $93,080,643$ & 270 & $\$ 27,000,000$ & $29 \%$ \\
90,000 & $1,440,000$ & $104,715,723$ & 300 & $\$ 30,000,000$ & $29 \%$ \\
100,000 & $1,600,000$ & $116,350,804$ & 330 & $\$ 33,000,000$ & $28 \%$ \\
\hline
\end{tabular}

\section{Conclusion and Future Work}

We observe a high degree of efficacy of our practical and easy to implement V2G optimization algorithm in leveraging the storage capacity of PEVs to improve the load carrying capacity of a grid. Also, our proposed simulation framework offers a very pragmatic, flexible and efficient approach toward analyzing and quantifying the impact of PEVs on a grid with varying fleet sizes, battery characteristics, vehicle efficiencies, commuting patterns, etc. It also provides an effective mechanism to estimate any potential impact of PEVs on the load carrying capacity of intermittent renewable power sources like solar.

Using the framework, we also were able to compute the economic benefit of PEVs in terms of their contribution of additional load carrying capacity to the grid. For the geographical area we studied in this paper - New York City - the potential capacity benefits represent about $\$ 20$ million a year in economic value in the 2015-2017 timeframe. This is about a third of the projected annualized cost of the PEV batteries. This benefit can potentially be shared between the grid operator or utility, the PEV owners, and any other stakeholders in the value chain. This benefit is on top of other benefits attributable to PEVs including emission reductions, energy security, etc.

We also observed in our work that incremental benefit in ELCC attributable to V2G goes down as installed solar capacity is increased. We have devised and simulated an effective approach of mitigating this effect via temporal spreading of PEV commute profiles. We have proposed a new metric for computing the ELCC efficiency of various commute profiles and demonstrated that the metric can be improved by up to $72 \%$ through suitable temporal shaping of commute profiles.

Potential future work would include running the simulation framework for other geographical areas with their own load, generation, and commute characteristics, PEV fleet size projections, other makes and models of PEVs, and with data over longer periods of time to identify larger patterns and trends. It would also be beneficial to run the framework for other intermittent renewable energy sources, most notably wind, and compare the results with the findings for solar generation reported in this paper. Another useful avenue of study would be to explore the feasibility and efficacy of various ways of motivating employers and commuters to spread out the commute times including sharing some of the economic benefits from such temporal adjustments between the grid operators, utilities, employers and commuters.

\section{References}

[1] E. Ungar, K. Fell, "Plug In, Turn On, and Load Up”, IEEE Power and Energy Magazine, Vol 8, Issue 3, May/June 2010.

[2] Solar Energy Industries Association and GTM Research, US Solar Market Insight Report 2011 http://www.seia.org/research-resources/us-solar-market-insight-report-201 1-year-review

[3] L.L. Garver, Effective Load carrying Capability of Generating Units, IEEE Transactions, Power Apparatus and Systems. Vol. Pas-85, No.8, 1966. [4] 3 Numbers to Rate Volt's Fuel Economy, www.nytimes.com/2010/11/25/business/25volt.html

[5] S.V. Chakraborty, S.K. Shukla, J. Thorp, “A Detailed Analysis of the Effective-Load-Carrying-Capacity Behavior of Plug-in Electric Vehicles in the Power Grid”, IEEE PES Conference on Innovative Smart Grid Technologies 2012, Washington, D.C.

[6] W. Kempton, and S.E. Letendre, Electric vehicles as a new power source for electric utilities. Transportation Research Part D 2 3, 1997, 157-175.

[7] P. Lombardi, P. Vasquez, Z. A. I. Styczynski, "Plug-in electric vehicles as storage devices within an Autonomous power system. Optimization issue”, IEEE PowerTech, 2009, Bucharest.

[8] H. Lund and W. Kempton, "Integration of renewable energy into the transport and electricity sectors through V2G”, Energy policy, Elsevier Ltd., 2008.

[9] A. Y. Saber and G. K. Vinayagamoorthy, "Unit Commitment with Vehicle-to-Grid using Partical Swarm Optimization”, IEEE PowerTech, 2009, Bucharest.

[10] P. B. Evens, S. Kuloor, "Impact of Plug-in Vehicles and Distributed Storage on Electric Power Delivery Networks”, IEEE Vehicle Power and Propulsion Conference, 2009. Dearborn, MI.

[11] E. Larsen, D. Chandrashekhara, J. Østergård, "Electric Vehicles for Improved Operation of Power Systems with High Wind Power Penetration”, IEEE Energy2030, 2008, Atlanta, GA. 
[12] A.Y. Saber, G.K. Venayagamoorthy, "Optimization of Vehicle-to-Grid Scheduling in Constrained Parking Lots", IEEE Power General Society General Meeting, 2009, Calgary, Canada.

[13] J. A. Peças Lopes, P. M. Rocha Almeida, F. J. Soares, "Using Vehicleto-Grid to Maximize the Integration of Intermittent Renewable Energy Resources in Islanded Electric Grids", International Conference on Clean Electrical Power, 2009, Ischia - Italy.

[14] W. Kempton and J. Tomic, "Vehicle-to-grid power fundamentals: Calculating capacity and net revenue," Journal of Power Sources, Vol. 144, No. 1, 268-279, June 2005.

[15] K. Parks, P. Denholm, T. Markel, "Costs and Emissions Associated with Plug-in Hybrid Electric Vehicle Charging in the Xcel Energy Colorado Service Territory", Technical Report, National Renewable Energy Laboratory, May 2007.

[16] S.-L. Andersson, A. Elofsson, M. Galus, L. Gransson, S. Karlsson, F. Johnsson, and G. Andersson, 2010 "Plug-in Hybrid Electric Vehicles as Regulating Power Providers: Case Studies of Sweden and Germany,” Energy Policy, vol. 38, no. 6, $2751-2762$.

[17] C. Binding, D. Gantenbein, B. Jansen, O. Sundström, P. B. Andersen, F. Marra, B. Poulsen, and C. Træhold, "Electric Vehicle Fleet Integration in the Danish EDISON Project - A Virtual Power Plant on the Island of Bornholm," IEEE PESGeneral Meeting, 2010, Minneapolis, MN.

[18] J. R. Pillai, B. Bak-Jensen, "Integration of Vehicle-to-Grid in the Western Danish Power System”, IEEE Transactions on Sustainable Energy, Vol. 2, No. 1, January 2011.

[19] A. Brooks, E. Lu, D. Reicher, C. Spirakis, B. Weihl, "Demand Dispatch - Using Real-Time Control of Demand to Help Balance Generation and Load”, IEEE Power and Energy Magazine, Vol 8, Issue 3, May/June 2010.

[20] A.B. Pedersen, B. Poulsen, E.B. Hauksson, C. Traeholt, P.B. Andersen, D. Gantenbein, "Facilitating a Generic Communication Interface to Distributed Energy Sources", $1^{\text {st }}$ IEEE International Conference on Smart Grid Communications, 2010, Gaithersburg, MD.

[21] B. Jansen, C. Binding, O. Sundstrom, D. Gantenbein, "Architecture and Communication of an Electric Vehicle Virtual Power Plant", $1^{\text {st }}$ IEEE International Conference on Smart Grid Communications, 2010, Gaithersburg, MD.

[22] M. Amelin, L. Soder, "Taking Credit - The Impact of Wind Power on Supply Adequacy - Experience from the Swedish Market”, IEEE Power and Energy Magazine, Vol 8, Issue 5, Sep/Oct 2010.

[23] M. Amelin, "Comparison of Capacity Credit Calculation Methods for Conventional Power Plants and Wind Power", IEEE Transactions on Power Systems, Vol. 24, No. 2, May 2009.

[24] A. Keane, M. Milligan, C. Dent, B. Hasche, L. Soder, M. O’Malley, Task Force on the Capacity Value of Wind Power, IEEE Power and Energy Society, "Capacity Value of Wind Power", IEEE Transactions on Power Systems, Vol. 26, No. 2, May 2011.

[25] R. Perez, R. Margolis, M. Kmiecik, M. Schwab, M. Perez, "Update: Effective Load-Carrying Capability of Photovoltaics in the United States", National Renewable Energy Laboratory, June 2006

[26] New York ISO: Markets \& Operations - Load Data, http://www.nyiso.com/public/markets_operations/market_data/load_data/i ndex.jsp

[27] New York State Reliability Council (NYSRC) Installed Reserve Margin Study, http://www.nysrc.org/NYSRC_NYCA_ICR_Reports.asp

[28] National Climatic Data Center (NCDC) - hourly solar radiation data, ftp://ftp.ncdc.noaa.gov/pub/data/uscrn/products/hourly02/

[29] General Motors Chevy Volt - Official Site, http://www.chevrolet.com/volt/

[30] Federal Energy Regulatory Commission (FERC), Market Oversight Data for New York ISO Capacity Auction Prices: http://www.ferc.gov/market-oversight/mkt-electric/new-york/elec-ny-rto-c ap-pr.pdf

[31] S.B. Peterson, J. Apt, J.F. Whitacre, "Lithium-ion Battery Cell Degradation Resulting from Realistic Vehicle and Vehicle-to-Grid Utilization”, Journal of Power Sources, Vol 195, 2010.

[32] E. Wood, M. Alexander, T. H. Bradley, "Investigation of Battery End-of-Life Conditions for Plug-in Hybrid Electric Vehicles", Journal of Power Sources, Vol 196, 2011.

\section{Biographies}

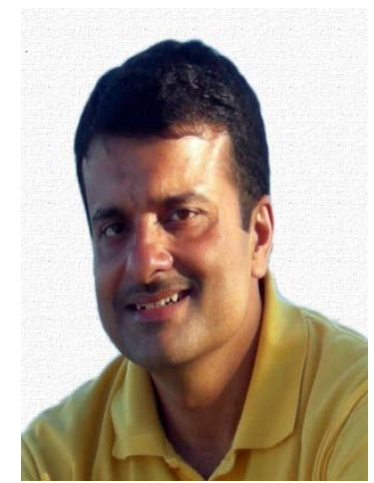

Soumyo V. Chakraborty is a graduate student in the Department of Electrical and Computer Engineering at Virginia Polytechnic Institute and State University. His research interests include renewable energy sources and their integration into power systems, smart grids, and the economics of renewable technologies. He has an MS in computer science from Rutgers University and an MBA from the Wharton School of the University of Pennsylvania.

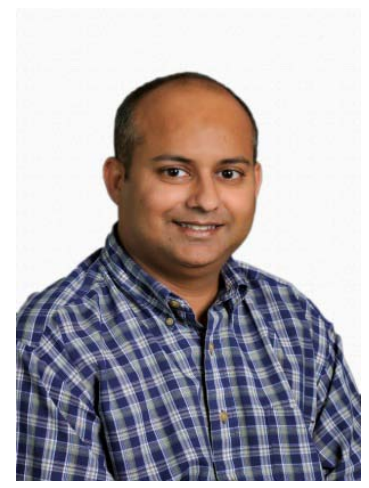

Sandeep K. Shukla is a professor in the Department of Electrical and Computer Engineering at Virginia Polytechnic Institute and State University. He is an associate editor of IEEE Transactions on Computers, IEEE Design \& Test, and IEEE Embedded Systems letters. He was awarded the PECASE in 2004, and Humboldt Foundation's Bessel award in 2008 .

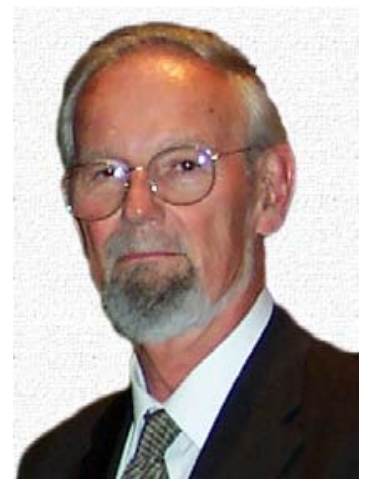

James Thorp is an emeritus professor in the Department of Electrical and Computer Engineering at Virginia Polytechnic Institute and State University. Prof. Thorp was an associate editor for IEEE Transactions on Circuits and Systems from 1985 to 1987. $\mathrm{He}$ is a member of the National Academy of Engineering, a member of the IEEE Power System Relaying Committee, CIGRE, Eta Kappa Nu, Tau Beta Pi, and Sigma Xi. He was also co-recipient of the Benjamin Franklin Medal in 2008. 